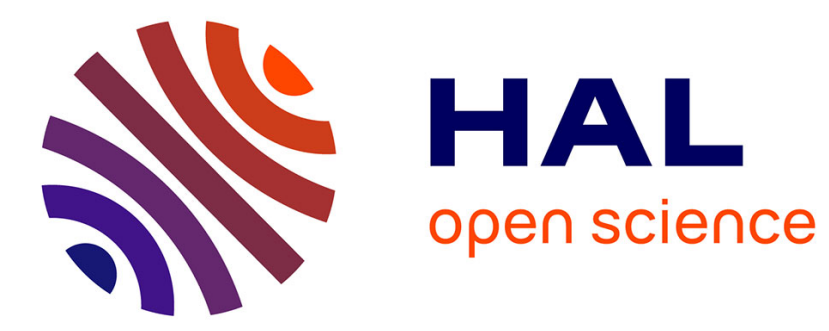

\title{
Can incremental expansions cope with high-order coupled-cluster contributions?
}

Hermann Stoll

\section{To cite this version:}

Hermann Stoll. Can incremental expansions cope with high-order coupled-cluster contributions? Molecular Physics, 2010, 108 (03-04), pp.243-248. 10.1080/00268970903313675 . hal-00580700

\section{HAL Id: hal-00580700 https://hal.science/hal-00580700}

Submitted on 29 Mar 2011

HAL is a multi-disciplinary open access archive for the deposit and dissemination of scientific research documents, whether they are published or not. The documents may come from teaching and research institutions in France or abroad, or from public or private research centers.
L'archive ouverte pluridisciplinaire HAL, est destinée au dépôt et à la diffusion de documents scientifiques de niveau recherche, publiés ou non, émanant des établissements d'enseignement et de recherche français ou étrangers, des laboratoires publics ou privés. 


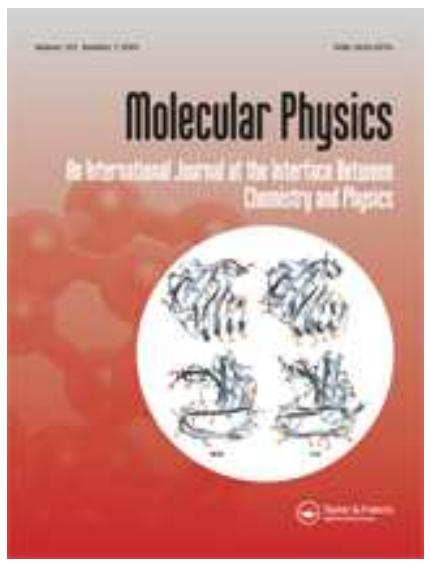

\section{Can incremental expansions cope with high-order coupled- cluster contributions?}

\begin{tabular}{|c|c|}
\hline Journal: & Molecular Physics \\
\hline Manuscript ID: & TMPH-2009-0230.R1 \\
\hline Manuscript Type: & Special Issue Paper - In honour of Prof Werner 60th birthday \\
\hline $\begin{array}{l}\text { Date Submitted by the } \\
\text { Author: }\end{array}$ & 26-Aug-2009 \\
\hline Complete List of Authors: & Stoll, Hermann; Theoretische Chemie \\
\hline Keywords: & ab-initio, coupled-cluster, increments \\
\hline \multicolumn{2}{|c|}{$\begin{array}{l}\text { Note: The following files were submitted by the author for peer review, but cannot be converted } \\
\text { to PDF. You must view these files (e.g. movies) online. }\end{array}$} \\
\hline paper_mod.tex & \\
\hline
\end{tabular}

\section{今) ScholaroNE \\ Manuscript Central}




\title{
Can incremental expansions cope with high-order
} coupled-cluster contributions?

\author{
Hermann Stoll* \\ Institut für Theoretische Chemie, Universität Stuttgart \\ D-70550 Stuttgart, Germany
}

\begin{abstract}
:
Local-correlation incremental expansions have been used for the evaluation of high-order coupled-cluster corrections to atomization energies, in exploratory calculations for small molecules. Expansion up to (at least) 3-body terms seems to be necessary in terms of localized orbitals, within the occupied space, while truncation after 2-body inter-atomic terms appears to be sufficient in the virtual space.
\end{abstract}

\footnotetext{
*e-mail: stoll@theochem.uni-stuttgart.de
} 


\section{Introduction}

An important feature of quantum-chemical wavefunction-based ab-initio methods is their ability to allow for a systematic improvement towards the exact solution of the Schrödinger equation. The price to pay for that is the steep increase of computational effort, both with respect to the size of the one-particle basis set and the level of electron correlation treatment. For example, the computational effort for a state-of-the-art coupled-cluster calculation with single and double excitations and perturbative account of triples $(\operatorname{CCSD}(\mathrm{T}))$ rises as $n_{\mathrm{occ}}^{3} n_{\mathrm{virt}}^{4}$, where $n_{\text {occ }}$ and $n_{\text {virt }}$ are the number of occupied and virtual orbitals, respectively. Going to a higher-order coupled-cluster treatment, i.e., including triple excitations beyond perturbation theory (CCSDT) or additional quadruples (CCSDTQ) leads to computation times $\sim n_{\mathrm{occ}}^{3} n_{\mathrm{virt}}^{5}$ or $\sim n_{\text {occ }}^{4} n_{\text {virt }}^{6}$.

Most fruitful for overcoming the computational bottleneck and making large molecules accessible to an accurate ab-initio treatment have been the ideas of local correlation and of explicitly correlated wavefunctions. These ideas, which have been pioneered by Pulay and Saebo $[1,2,3]$ and by Kutzelnigg and Klopper $[4,5,6]$, have been in the focus of the scientific work of Hans-Joachim Werner for nearly two decades, see e.g. [7, 8, 9] and references therein. He succeeded in developing correlation methods up to $\operatorname{CCSD}(\mathrm{T})$ which linearly scale with molecular size and allow for inter-electronic terms in the wavefunction thereby greatly reducing the number of basis functions per atom.

The present work is meant as a preliminary exploration on whether similar ideas, local correlation in particular, may be useful for evaluating correlation corrections beyond $\operatorname{CCSD}(\mathrm{T})$. Such corrections are usually quite small, with contributions in the $\mathrm{kJ} / \mathrm{mol}$ regime, but have proven to be important for accurate work on atomization or excitation energies, see e.g. $[10,11,12,13]$. The approach used in the present work is the incremental scheme (see, e.g., $[14,15])$ which consists in a many-body expansion of the correlation energy in terms of local corrections. After giving some computational details in Sect. 2 this approach is applied to small molecules in the following two sections, with an expansion in terms of occupied localized orbitals in Sect. 3 and an expansion in terms of local corrections in the virtual space in Sect. 4. Some conclusions are drawn in Sect. 5 . 


\section{Computational details}

All calculations of the present work have been performed using the Molpro suite of ab-initio programs developed by Werner, Knowles and co-workers [16, 17, 18, 19], and the multireference coupled-cluster (MRCC) code by Kállay [20, 21, 22] interfaced to Molpro. Basis sets are taken from the series of correlation-consistent polarized valence $n$-zeta (cc-pVnZ) basis sets of Dunning and co-workers [23], or from the corresponding augmented (aug-cc-pVnZ) sets [24]. Due to the high computational effort for the higher-order coupled-cluster treatment (CCSDT and CCSDTQ), rather small basis sets of double-zeta or triple-zeta quality had to be used for evaluating the non-perturbative triples and quadruples contributions which are at the focus of the present work. (For the sake of comparison, we also give contributions from singles/doubles and perturbative triples using the same basis sets, in Sects. 3 and 4, although for an accurate determination of atomization energies larger basis sets would be needed at the CCSD and $\operatorname{CCSD}(\mathrm{T})$ levels.)

For localizing orbitals, the Foster-Boys criterion [25] has been applied. One typical step of the incremental scheme of Sect. 3 consists in correlating a group of localized orbitals (LMOs) while keeping all the other LMOs as frozen. Before correlating such a group in, e.g., CCSD(T) calculations, the active (i.e., non-frozen) orbitals were canonicalized among each other using the multi-configuration self-consistent-field (MCSCF) facility of Molpro, i.e., pseudo-canonical orbitals were generated.

The incremental scheme involves a many-body expansion for the correlation energy of the system, of the form

$$
\begin{gathered}
E^{\mathrm{corr}}=E_{0}^{\mathrm{corr}}+\sum_{A} \epsilon_{A}+\frac{1}{2 !} \sum_{A B} \epsilon_{A B}+\frac{1}{3 !} \sum_{A B C} \epsilon_{A B C}+\ldots \\
\epsilon_{A}=E_{A}^{\mathrm{corr}} \\
\epsilon_{A B}=E_{A B}^{\mathrm{corr}}-\epsilon_{A}-\epsilon_{B} \\
\epsilon_{A B C}=E_{A B C}^{\mathrm{corr}}-\epsilon_{A B}-\epsilon_{B C}-\epsilon_{C A}-\epsilon_{A}-\epsilon_{B}-\epsilon_{C}
\end{gathered}
$$

Here, $E_{0}^{\text {corr }}$ is a reference correlation energy (to be specified below, see Sects. 3 and 4). A, $\mathrm{B}, \mathrm{C}$ denote individual atoms or LMOs, and $\mathrm{AB}, \mathrm{ABC}$ refer to groups of atoms or LMOs. 
$E_{A}^{\mathrm{corr}}, E_{A B}^{\mathrm{corr}}, E_{A B C}^{\mathrm{corr}}$ are correlation-energy increments, with respect to $E_{0}^{\mathrm{corr}}$, when improving the correlation treatment locally at A, AB, or ABC (see Sects. 3 and 4 for more details). Finally, the $\epsilon_{A}, \epsilon_{A B}, \epsilon_{A B C}$ are one-body increments and non-additive two-body and threebody corrections, respectively. In the following, we consider differential quantities, e.g. $\Delta \epsilon_{A}$, $\Delta \epsilon_{A B}, \Delta \epsilon_{A B C}$, which arise when enhancing the correlation level for the treatment of the system, i.e., restricting the $\mathrm{CC}$ calculations to singles and doubles, or considering changes due to perturbative triples, or triples beyond $\operatorname{CCSD}(\mathrm{T})$, or quadruples beyond CCSDT.

\section{Incremental expansion in occupied space}

Here, the reference level is SCF ( $\left.E_{0}^{\text {corr }}=0\right)$, The indices A, B, C denote Foster-Boys localized orbitals, and the correlated orbital space for evaluating $E_{A}^{\text {corr }}, E_{A B}^{\text {corr }}, E_{A B C}^{\text {corr }}$ is restricted to the LMOs indicated, i.e., only 1, 2, or 3 occupied orbitals are correlated simultaneously for a given one-body, two-body, or three-body increment.

As a first example, we consider the $\mathrm{CH}_{4}$ molecule (with $r_{\mathrm{C}-\mathrm{H}}=1.102 \AA$ ), with four equivalent LMOs describing C-H bonds. Results with cc-pVDZ basis set are listed in Table 1. As known from previous work $[14,15]$, CCSD correlation energies rapidly converge to the full CCSD value, within an incremental expansion. While one-body terms are clearly insufficient, yielding only $\sim 60 \%$ of the full value due to the lack of inter-orbital correlation, two-body terms are already quite accurate, overshooting the full value by $\sim 3 \%$, and inclusion of three-body terms leads to an accuracy of better than $0.1 \%$. As may be expected, the convergence characteristics somewhat deteriorate when considering the contributions of perturbative triples. Clearly, there are no one-body terms here at all, the two-body terms amount to $\sim 90 \%$ of the full value, and the accuracy reached at the three-body level is $\sim 2 \%$. For post-CCSD $(\mathrm{T})$ triples contributions (T-(T) in Table 1) and quadruples (Q), i.e., for increasing number of LMOs simultaneously involved, the two-body level of the incremental expansion becomes clearly insufficient, with deviations from the full results by factors of $\sim 2$, but three-body terms lead to an efficient improvement, with deviations of up to $3 \%$ only. Since the absolute deviations are $<0.04 \mathrm{~kJ} / \mathrm{mol}$, this seems to provide sufficient accuracy for most purposes. 
In order to check how sensitive the above findings are with respect to the choice of the orbitals used, let us adopt the worst case, i.e., the case of completely delocalized canonical orbitals (CMO), cf. Table 2. As may be expected, the leading terms in the incremental expansion go down in magnitude by up to factors of 2 , but the results reached at the three-body level still seem to be useful: accuracies around 1\% are reached for CCSD and (T) correlation energies, and of $7 \%$ and $13 \%(0.07 \mathrm{~kJ} / \mathrm{mol})$ for $\mathrm{T}-(\mathrm{T})$ and $\mathrm{Q}$ contributions, respectively.

Turning now to the discussion of the influence of the one-particle basis sets, let us consider results for $\mathrm{CH}_{4}$ with triple-zeta basis sets (cc-pVTZ), see Table 3. Compared to cc-pVDZ, the correlation energy naturally increases in magnitude, but in a different way for different excitation classes. CCSD yields the largest absolute enhancement, of course, but percentagewise the increase is largest for the perturbative triples (in $\operatorname{CCSD}(\mathrm{T})$ ). Post-CCSD $(\mathrm{T})$ triples (in CCSDT) and quadruples (in CCSDTQ) lead to an only moderate increase of 8\% (Q) or even a slight decrease $(\mathrm{T}-(\mathrm{T}))$. The performance of the many-body expansion turns out to be very similar to that for the smaller basis. At the three-body level, the contribution of double excitations is recovered with an accuracy of $<0.1 \%$, that of perturbative triples to $<2 \%$, while the accuracy is lower again for post-CCSD(T) triples (4\%) and quadruples (6\%). Still, the absolute errors in the latter cases are smaller than $0.05 \mathrm{~kJ} / \mathrm{mol}$.

A final check deals with the size of the molecule: Table 4 presents results for $\mathrm{C}_{2} \mathrm{H}_{6}$ (ccpVDZ basis, $r_{\mathrm{C}-\mathrm{C}}=1.544 \AA, r_{\mathrm{C}-\mathrm{H}}=1.102 \AA$ ). It is clear that truncation of the many-body expansion at a given order becomes more stringent with increasing number of subsystems. Still, the correlation contribution of double excitations is accurate to $\sim 0.1 \%$ at the threebody level, and that of perturbative triples to $\sim 2 \%$. However, post-CCSD(T) triples are overestimated by ca. $30 \%$ here! The total percentage error for the triples is not so very much larger for $\mathrm{C}_{2} \mathrm{H}_{6}(4 \%)$ than for $\mathrm{CH}_{4}(2 \%)$, but that of post-CCSD(T) triples is. Four-body terms are needed for reducing the error of the T-(T) contribution. Actually, four-body terms with 3 LMOs sharing a common atom are sufficient to bring the deviation down to $<1 \%$, with the largest effect coming from terms connecting the C-C bond with C-H LMOs on both sides. Further investigations are necessary in order to monitor the performance of the incremental expansion when further increasing the size of the molecules. Interestingly, the convergence 
of the many-body expansion seems to be more rapid for the full triple contribution, $\mathrm{T}$, than for the perturbative (T) one: the leading three-body increments (involving 3 LMOs with one atom in common) are by more than an order of magnitude smaller than the corresponding two-body ones, for both $\mathrm{D}$ and $\mathrm{T}$ contributions, while the factor is only $\sim 5$ for the $(\mathrm{T})$ ones. Anyway, the $\mathrm{Q}$ contribution in $\mathrm{C}_{2} \mathrm{H}_{6}$ is more similar in accuracy again to that in $\mathrm{CH}_{4}$; at the three-body level, it is by $6 \%$ off the full value.

Summarizing, we can say that incremental expansions in terms of localized orbitals form a computationally efficient way of calculating contributions of post-CCSD(T) triples and quadruples in coupled-cluster calculations. Since individual increments rapidly decrease with increasing distance between LMOs, the computational effort eventually increases only linearly with molecular size. However, at least three-body terms have to be included into the expansion in order to get meaningful results.

A decomposition of higher-order coupled-cluster contributions to atomization energies into bond increments has recently been suggested by Bakowies [13]. His suggestion differs from that of the present work in that the increments are taken from larger entities, i.e., the bond increment for $\mathrm{C}-\mathrm{H}$ is taken from a full calculation for $\mathrm{CH}_{4}$, that for $\mathrm{C}-\mathrm{C}$ is calculated from full calculations for $\mathrm{C}_{2} \mathrm{H}_{6}$ and $\mathrm{CH}_{4}$. Furthermore, only effective one-body (bond) terms are defined, i.e., two-body and three-body terms are set to zero, which somewhat obscures the importance of the latter found in the present work. Finally, the very different T-(T) contribution for C-H and C-C reported in Ref. [13] (-0.021 kcal/mol and $-0.221 \mathrm{kcal} / \mathrm{mol}$, respectively) is in contrast to our observation that one-, two-, and three-body increments involving C-C LMOs change only very little when comparing to the corresponding increments with C-C replaced by C-H LMOs; examples are the T-(T) contribution to the C-C/C-H and C-H/C-H two-body increments $\left(-1.06 \cdot 10^{-4}\right.$ a.u. vs. $-1.18 \cdot 10^{-4}$ a.u. $)$, or the C-C/C-H/C-H and $\mathrm{C}-\mathrm{H} / \mathrm{C}-\mathrm{H} / \mathrm{C}-\mathrm{H}$ three-body increments $\left(+6.7 \cdot 10^{-5}\right.$ a.u. vs. $+7 \cdot 3 \cdot 10^{-5}$ a.u. $)$.

\section{Incremental expansion in virtual space}

Here, the indices A, B, C in eqs. 1 - 4 denote atoms, and the incremental expansion proceeds by enlarging the virtual space atom by atom. Starting-point is the correlation energy $E_{0}^{\text {corr }}$ 
evaluated with a (small) reference basis set, and this energy is improved within $E_{A}^{\text {corr }}, E_{A B}^{\text {corr }}$, $E_{A B C}^{\text {corr }}$, by using a (larger) target basis set at one, two, or three atoms (A, $\mathrm{AB}$, or $\mathrm{ABC}$ ). Localization in occupied space is not necessary here, and the correlated occupied orbital space does not change within the expansion (being that of the full system).

Let us begin our examples with $\mathrm{CH}_{4}$ again and consider the incremental expansion for extending the basis set from cc-pVDZ to cc-pVTZ. As already mentioned, the perturbative triples contribution, $(\mathrm{T})$, in the coupled-cluster series is that which is mostly affected percentage-wise by the basis-set change (increasing by ca. 70\%). It is seen from Table 5 that it converges quite rapidly with the order of the incremental expansion, being by $14 \%$ off the cc-pVTZ results at the one-body level, and by only $\sim 1 \%$ at the two-body one. Similar accuracies, of $\sim 0.5 \%$ for doubles (D), of $<1 \%$ for the $\mathrm{T}-(\mathrm{T})$ and $\mathrm{Q}$ ones, are achieved at the two-body level for the other contributions. However, this is no stringent test of the incremental expansion for higher-order coupled-cluster methods, since the T-(T) and Q contributions change only very little (by <10\%) when going from cc-pVDZ to cc-pVTZ.

As a simple, but more significant test, we next consider the $\mathrm{C}$ atom, cf. Table 6 . Here, we extend the cc-pVDZ basis set shell by shell towards cc-pVTZ, i.e., the indices A,B,C are taken as shell indices $s, p, d, f$, and the basis functions of the smaller set are replaced step by step with the functions of the larger basis. For the $\mathrm{C}$ atom, the $\mathrm{T}-(\mathrm{T})$ and $\mathrm{Q}$ contributions increase by $78 \%$ and $45 \%$, respectively, when going from cc-pVDZ to cc-pVTZ. Here, the incremental expansion converges even faster than in the preceding example. The T-(T) and $\mathrm{Q}$ contributions are off the final (cc-pVTZ) result by $<4 \%$, when enlarging the basis set for only one shell at a time, and the changes at the two-body level (enlarging two shells simultaneously) mostly improve the $\mathrm{D},(\mathrm{T})$, and $\mathrm{T}-(\mathrm{T})$ contributions to an accuracy $<0.5 \%$.

Let us now turn to a more complex and demanding system. The $\mathrm{O}_{3}$ molecule is among those with the largest high-order coupled-cluster corrections of the molecules considered in Refs. $[11,12,13]$, due to its multi-reference character. Our results to be discussed in the following refer to the experimental geometry $\left(r_{\mathrm{O}-\mathrm{O}}=1.272 \AA, \theta=116.8^{0}\right)$. From Table 7 , it is seen that coupled-cluster calculations are sufficiently different for the $s p$ part of the cc-pVDZ basis set and the full cc-pVDZ set, but that the difference can be systematically 
and efficiently decreased with an incremental expansion. For the D and (T) values, even the one-body level is quite satisfactory, with deviations of $\sim 1 \%$. For the T-(T) contribution, on the other hand, which even changes sign between cc-pVDZ $(s p)$ and cc-pVDZ, two-body terms are necessary to reach an accuracy of $\sim 10 \%$. (For the $\mathrm{Q}$ contribution, the starting-point is nearer to the final result, and convergence is faster again.) It is interesting to note that inclusion of diffuse functions (aug-cc-pVDZ basis) significantly change the coupled-cluster correlation contributions, by $5 \%$ and $13 \%$ for D and (T), respectively, and even change sign for T-(T); such augmented basis sets have not been considered in Refs. $[11,12,13]$. In the same vein, as before, we consider an incremental expansion starting with the $s p$ part of the aug-cc-pVDZ basis and leading eventually to the full aug-cc-pVDZ set, cf. Table 8. As before, the convergence of the expansion is quite fast, with deviations from the full result of $0.1 \%$ for (T), and $13 \%$ for $\mathrm{T}-(\mathrm{T})$, at the two-body level; even one-body results are meaningful here, throughout.

As a final example, let us compare the (not fully satisfactory) incremental expansion for $\mathrm{C}_{2} \mathrm{H}_{6}$ of the previous section to a corresponding expansion in virtual (rather than occupied) space, extending the $s p$ part of the cc-pVDZ basis set to the full cc-pVDZ one, see Table 9. It turns out that, although the individual contributions change by up to a factor 2 , in the course of the expansion, the results are fully satisfactory at the two-body level, with deviations of $0.5 \%, 0.7 \%, 0.8 \%$, and $2.3 \%$, for $\mathrm{D},(\mathrm{T}), \mathrm{T}-(\mathrm{T})$, and $\mathrm{Q}$, respectively.

\section{Discussion and Conclusion}

Incremental expansions of correlation energies in terms of local entities inherently lead to a computational effort for evaluating these energies which scales linearly with the size of the system. This is true provided that correlation effects go to zero sufficiently fast with increasing distance between the entities. It is not strictly true for the case of the expansion in terms of virtual orbitals when starting with a non-minimal basis set, since the reference energy $E_{0}^{\text {corr }}$ of eq. 1 does not scale linearly; however, in principle, the starting point could just be a basis set consisting of the occupied SCF orbitals $\left(n_{v}=0\right)$. Of course, the systems considered in this paper are too small to enter such a linearly scaling regime. For $\mathrm{C}_{2} \mathrm{H}_{6}$, however, we 
can at least check the above assumption of the decrease of correlation contributions with distance: the $\mathrm{C}-\mathrm{H} / \mathrm{C}-\mathrm{H}$ two-body increment decreases by an order of magnitude when going from nearest-neighbour to second-nearest-neighbour LMO pairs, and this is not only true for the total increment, but also for its $\mathrm{D},(\mathrm{T}), \mathrm{T}-(\mathrm{T})$, and Q parts separately.

As a disadvantage, the introduction of local entities precludes the use of symmetry which is very helpful in higher-order coupled-cluster calculations. On the other hand, symmetry is not completely lost: it could be partially retained $\left(\mathrm{C}_{s}\right)$ for $\mathrm{O}_{3}$, e.g., and even with (partial) canonicalization the incremental scheme can still be expected to work (cf. Table 2). It would be perfectly possible, in principle, to merge symmetry-equivalent LMOs or atoms to a single entity for the incremental expansion.

From the viewpoint of computational effort, the expansion for the virtual space seems to be preferable over that for the occupied space, since a) no localization step is needed in the former case, b) truncation at the two-body level seems to be possible, while at least threebody terms are needed in the latter case, and c) the CPU time for standard coupled-cluster calculations rises more steeply with the number of virtual than occupied orbitals (see Sect. 1). However, it would also be possible to somehow combine the two types of expansions as suggested in previous work [15], i.e., to use good-quality basis sets only for those atoms which belong to the domain of the LMOs to be correlated. This would naturally fit into the local-correlation philosophy of Werner and co-workers. 


\section{References}

[1] P. Pulay, Chem. Phys. Lett. 100, 151 (1983).

[2] S. Saebø and P. Pulay, J. Chem. Phys. 86, 914 (1987).

[3] S. Saebø and P. Pulay, J. Chem. Phys. 88, 1884 (1988).

[4] W. Kutzelnigg and W. Klopper, J. Chem. Phys. 94, 1985 (1991).

[5] V. Termath, W. Klopper, and W. Kutzelnigg, J. Chem. Phys. 94, 2002 (1991).

[6] W. Klopper and W. Kutzelnigg, J. Chem. Phys. 94, 2020 (1991).

[7] H.-J. Werner, J. Chem. Phys. 129, 101103 (2008).

[8] G. Knizia, T.B. Adler, and H.-J. Werner, J. Chem. Phys. 130, 054104 (2009).

[9] T.B. Adler, F.R. Manby, and H.-J. Werner, J. Chem. Phys. 130, 054106 (2009).

[10] N.B. Balabanov and K.A. Peterson, J. Chem. Phys. 125, 074110 (2006).

[11] D. Feller, K.A. Peterson, and D.A. Dixon, J. Chem. Phys. 129, 204105 (2008).

[12] W. Klopper, B. Ruscic, D.P. Tew, F.A. Bischoff, and S. Wolfsegger, Chem. Phys. 356, $14(2009)$.

[13] D. Bakowies, J. Chem. Phys. 130, 144113.

[14] H. Stoll, Phys. Rev. B 46, 6700 (1992).

[15] H. Stoll, B. Paulus, and P. Fulde, J. Chem. Phys 123, 144108 (2005).

[16] MOLPRO, version 2008.1, a package of ab initio programs, H.-J. Werner, P.J. Knowles, R. Lindh, F.R. Manby, M. Schütz, P. Celani, T. Korona, A. Mitrushenkov, G. Rauhut, T.B. Adler, R.D. Amos, A. Bernhardsson, A. Berning, D.L. Cooper, M.J.O. Deegan, A.J. Dobbyn, F. Eckert, E. Goll, C. Hampel, G. Hetzer, T. Hrenar, G. Knizia, C. Köppl, Y. Liu, A.W. Lloyd, R.A. Mata, A.J. May, S.J. McNicholas, W. Meyer, M.E. Mura, A. Nicklass, P. Palmieri, K. Pflüger, R. Pitzer, M. Reiher, U. Schumann, H. Stoll, A.J. Stone, R. Tarroni, T. Thorsteinsson, M. Wang, and A. Wolf, see http://www.molpro.net. 
[17] C. Hampel, K. Peterson, and H.-J. Werner, Chem. Phys. Lett. 190, 1 (1992).

[18] M.J.O. Deegan and P.J. Knowles, Chem. Phys. Lett. 227, 321 (1994).

[19] P.J. Knowles, C. Hampel, and H.-J. Werner, J. Chem. Phys. 99, 5219 (1993); Erratum: J. Chem. Phys. 112, 3106 (2000).

[20] MRCC, a string-based quantum chemical program suite written by M. Kállay, see http://www.mrcc.hu.

[21] M. Kállay and P.R. Surján, J. Chem. Phys. 115, 2945 (2001).

[22] M. Kállay, P.G. Szalay, and P.R. Surján, J. Chem. Phys. 117, 980 (2002).

[23] T.H. Dunning, Jr., J. Chem. Phys. 90 (1989) 1007.

[24] R.A. Kendall, T.H. Dunning, Jr., and R.J. Harrison, J. Chem. Phys. 96 (1992) 6796.

[25] J.M. Foster and S.F. Boys, Rev. Mod. Phys. 32, 296 (1960). 
Table 1: Contributions (a.u.) of doubles, D, perturbative triples, (T), full minus perturbative triples, T-(T), and quadruples, Q, to the coupled-cluster correlation energies of $\mathrm{CH}_{4}$ $\left(r_{\mathrm{C}-\mathrm{H}}=1.102 \AA\right.$, cc-pVDZ basis, Foster-Boys LMO); incremental expansion in terms of occupied orbitals, in parentheses: contributions to atomization energies

\begin{tabular}{|c|c|c|c|c|}
\hline & 1-body & 2-body & 3-body & full \\
\hline D & -0.118863 & -0.190762 & -0.185314 & $-0.185472(-0.108559)$ \\
$(\mathrm{T})$ & 0. & -0.003357 & -0.003836 & $-0.003772(-0.002795)$ \\
$\mathrm{T}-(\mathrm{T})$ & 0. & -0.000712 & -0.000413 & $-0.000400(-0.000076)$ \\
$\mathrm{Q}$ & 0. & -0.000064 & -0.000151 & $-0.000151(-0.000121)$ \\
\hline
\end{tabular}

Table 2: Contributions (a.u.) of doubles, D, perturbative triples, (T), full minus perturbative triples, T-(T), and quadruples, $\mathrm{Q}$, to the coupled-cluster correlation energies of $\mathrm{CH}_{4}$ $\left(r_{\mathrm{C}-\mathrm{H}}=1.102 \AA\right.$, cc-pVDZ basis, canonical MO); incremental expansion in terms of occupied orbitals, in parentheses: contributions to atomization energies

\begin{tabular}{|c|c|c|c|c|}
\hline & 1-body & 2-body & 3-body & full \\
\hline D & -0.064963 & -0.192537 & -0.184728 & $-0.185472(-0.108559)$ \\
$(\mathrm{T})$ & 0. & -0.002794 & -0.003820 & $-0.003772(-0.002795)$ \\
$\mathrm{T}-(\mathrm{T})$ & 0. & -0.000525 & -0.000429 & $-0.000400(-0.000076)$ \\
$\mathrm{Q}$ & 0. & -0.000032 & -0.000131 & $-0.000151(-0.000121)$ \\
\hline
\end{tabular}




\begin{tabular}{|c|c|c|c|c|}
\hline & 1-body & 2-body & 3-body & full \\
\hline $\mathrm{D}$ & -0.137088 & -0.224347 & -0.218738 & $-0.218880(-0.127192)$ \\
$(\mathrm{T})$ & 0. & -0.005430 & -0.006481 & $-0.006369(-0.004217)$ \\
$\mathrm{T}-(\mathrm{T})$ & 0. & -0.000806 & -0.000413 & $-0.000396(+0.000182)$ \\
$\mathrm{Q}$ & 0. & -0.000078 & -0.000172 & $-0.000162(-0.000120)$ \\
\hline
\end{tabular}

Table 3: Contributions (a.u.) of doubles, D, perturbative triples, (T), full minus perturbative triples, T-(T), and quadruples, $\mathrm{Q}$, to the coupled-cluster correlation energies of $\mathrm{CH}_{4}$ $\left(r_{\mathrm{C}-\mathrm{H}}=1.102 \AA\right.$, cc-pVTZ basis, Foster-Boys LMO); incremental expansion in terms of occupied orbitals, in parentheses: contributions to atomization energies

Table 4: Contributions (a.u.) of doubles, D, perturbative triples, (T), full minus perturbative triples, T-(T), and quadruples to the coupled-cluster correlation energies of $\mathrm{C}_{2} \mathrm{H}_{6}$ $\left(r_{\mathrm{C}-\mathrm{H}}=1.102 \AA, r_{\mathrm{C}-\mathrm{C}}=1.544 \AA\right.$, cc-pVDZ basis, Foster-Boys LMO); incremental expansion in terms of occupied orbitals, in parentheses: contributions to atomization energies

\begin{tabular}{|c|c|c|c|c|}
\hline & 1-body & 2-body & 3-body & full \\
\hline $\mathrm{D}$ & -0.204644 & -0.352865 & -0.340752 & $-0.340352(-0.186526)$ \\
$(\mathrm{T})$ & 0. & -0.006852 & -0.008595 & $-0.008420(-0.006466)$ \\
$\mathrm{T}-(\mathrm{T})$ & 0. & -0.001446 & -0.000830 & $-0.000639(+0.000008)$ \\
$\mathrm{Q}$ & 0. & -0.000124 & -0.000367 & $-0.000389(-0.000330)$ \\
\hline
\end{tabular}


Table 5: Contributions (a.u.) of doubles, D, perturbative triples, (T), full minus perturbative triples, T-(T), and quadruples, Q, to the coupled-cluster correlation energies of $\mathrm{CH}_{4}$ $\left(\mathrm{r}_{\mathrm{C}-\mathrm{H}}=1.102 \AA\right)$; incremental expansion in terms of cc-pVDZ $\rightarrow$ cc-pVTZ basis-set extension

\begin{tabular}{|c|c|c|c|c|c|}
\hline & start & 1-body & 2-body & 3-body & full \\
\hline D & -0.185472 & -0.229171 & -0.217986 & -0.218617 & -0.218880 \\
$(\mathrm{~T})$ & -0.003772 & -0.007261 & -0.006285 & -0.006342 & -0.006369 \\
$\mathrm{~T}-(\mathrm{T})$ & -0.000400 & -0.000412 & -0.000396 & -0.000394 & -0.000396 \\
$\mathrm{Q}$ & -0.000151 & -0.000145 & -0.000161 & -0.000171 & -0.000162 \\
\hline
\end{tabular}

Table 6: Contributions (a.u.) of doubles, D, perturbative triples, (T), full minus perturbative triples, T-(T), and quadruples, $\mathrm{Q}$, to the coupled-cluster correlation energies of the $\mathrm{C}$ atom $\left({ }^{3} \mathrm{P}\right.$ ground state); incremental expansion in terms of cc-pVDZ $\rightarrow$ cc-pVTZ basis-set extension, see text

\begin{tabular}{|c|c|c|c|c|c|}
\hline & start & 1-body & 2-body & 3-body & full \\
\hline D & -0.076913 & -0.091427 & -0.091670 & -0.091685 & -0.091688 \\
$(\mathrm{~T})$ & -0.000977 & -0.002039 & -0.002159 & -0.002152 & -0.002152 \\
$\mathrm{~T}-(\mathrm{T})$ & -0.000324 & -0.000566 & -0.000578 & -0.000578 & -0.000578 \\
$\mathrm{Q}$ & -0.000030 & -0.000041 & -0.000044 & -0.000042 & -0.000043 \\
\hline
\end{tabular}


Table 7: Contributions (a.u.) of doubles, D, perturbative triples, (T), full minus perturbative triples, $\mathrm{T}-(\mathrm{T})$, and quadruples, $\mathrm{Q}$, to the coupled-cluster correlation energies of $\mathrm{O}_{3}$ $\left(\mathrm{r}_{\mathrm{O}-\mathrm{O}}=1.272 \AA, \theta=116.8^{\circ}\right)$; incremental expansion in terms of cc-pVDZ $(s p) \rightarrow$ cc-pVDZ basis-set extension

\begin{tabular}{|c|c|c|c|c|}
\hline & start & 1-body & 2-body & full \\
\hline D & -0.437276 & -0.610871 & -0.610907 & -0.610757 \\
$(\mathrm{~T})$ & -0.026033 & -0.032653 & -0.033080 & -0.033103 \\
$\mathrm{~T}-(\mathrm{T})$ & +0.000346 & -0.000302 & -0.000130 & -0.000119 \\
$\mathrm{Q}$ & -0.004968 & -0.005282 & -0.005434 & -0.005431 \\
\hline
\end{tabular}

Table 8: Contributions (a.u.) of doubles, D, perturbative triples, (T), full minus perturbative triples, $\mathrm{T}-(\mathrm{T})$, and quadruples, $\mathrm{Q}$, to the coupled-cluster correlation energies of $\mathrm{O}_{3}$ $\left(\mathrm{r}_{\mathrm{O}-\mathrm{O}}=1.272 \AA, \theta=116.8^{\circ}\right)$; incremental expansion in terms of aug-cc-pVDZ $(s p) \rightarrow$ aug-ccpVDZ basis-set extension

\begin{tabular}{|c|c|c|c|c|}
\hline & start & 1-body & 2-body & full \\
\hline $\mathrm{D}$ & -0.446472 & -0.652964 & -0.641653 & -0.641039 \\
$(\mathrm{~T})$ & -0.027391 & -0.037359 & -0.037585 & -0.037546 \\
$\mathrm{~T}-(\mathrm{T})$ & +0.000564 & +0.000414 & +0.000416 & +0.000480 \\
\hline
\end{tabular}


Table 9: Contributions (a.u.) of doubles, D, perturbative triples, (T), full minus perturbative triples, T-(T), and quadruples, $Q$, to the coupled-cluster correlation energies of $\mathrm{C}_{2} \mathrm{H}_{6}$ $\left(r_{\mathrm{C}-\mathrm{H}}=1.102 \AA, r_{\mathrm{C}-\mathrm{C}}=1.544 \AA\right)$; incremental expansion in terms of cc-pVDZ $(s p) \rightarrow$ cc-pVDZ basis-set extension

\begin{tabular}{|c|c|c|c|c|}
\hline & start & 1-body & 2-body & full \\
\hline $\mathrm{D}$ & -0.217112 & -0.363065 & -0.342045 & -0.340352 \\
$(\mathrm{~T})$ & -0.003917 & -0.008090 & -0.008481 & -0.008420 \\
$\mathrm{~T}-(\mathrm{T})$ & -0.000402 & -0.000719 & -0.000644 & -0.000639 \\
$\mathrm{Q}$ & -0.000211 & -0.000428 & -0.000380 & -0.000389 \\
\hline
\end{tabular}

